\title{
Advanced Portable Preterm Baby Incubator
}

\author{
M. Shaib ${ }^{1}$, M. Rashid ${ }^{1}$, L. Hamawy ${ }^{1}$, M. Arnout ${ }^{1}$, I. El Majzoub ${ }^{2}$ and A. J. Zaylaa ${ }^{1,3, *}$ \\ ${ }^{1}$ Departments of Biomedical and Electrical Engineering, Lebanese International University, Lebanon \\ 2 American University of Beirut Medical Center, Lebanon \\ ${ }^{3}$ Neuroscience Research Center, Faculty of Medical Sciences, Lebanese University, Lebanon \\ * Corresponding Author
}

\begin{abstract}
Nearly 20 million premature and Low Birth Weight infants are born each year in developing countries, 4 million die within their first month. These deaths occur due to the unavailability or unreliability of traditional incubators. Moreover, although Telemedicine is helpful in rural areas, the shortage of healthcare providers have made it inaccessible in both basic healthcare. Thereby, traditional preterm baby and low-birth weight incubators and therapeutic techniques lack Telemedicine communication and feedback. The aim of our project is to develop an advanced portable and wireless-base incubator. We tend to provide an affordable, feasible, patient friendly and reliable premature baby incubator especially in low-income countries. The project focuses on the premature babies in the third trimester of pregnancy. The design is based on Wi-Fi and infrared technologies that measure the essential parameters that must be controlled for preemies. These parameters include the heart rate, oxygen level in the blood and temperature. Results showed the advanced design building blocks. The response of the generated power-voltage proves that the power can be regulated by the voltage. The thermal isolation can decrease the thermal lose and increase the time needed to drop temperature 6 times. In the room temperature of premature infant, $20^{\circ} \mathrm{C}$ and $45^{\circ} \mathrm{C}$, the resistance decreases from $12.69 \mathrm{k} \Omega$ to $4.82 \mathrm{k} \Omega$. The voltage and the temperature were inversely proportional. The heaters were efficient when tested on water. One of the major advantages of the device is that it surpasses existing techniques. As a future prospect more electronic components needs to be tested and features needs to be extracted.
\end{abstract}

Index Terms-Premature Baby, Advanced Incubator, Infrared Technology, Telemedicine, Diagnosis, Emergency, Therapy.

\section{INTRODUCTION}

Preterm delivery is defined as the occurrence of birth at less than 37 weeks of the gestation period [1]. In 2015, the World Health Organization (WHO) estimated that in 1 million among 15 million of preemies do not survive due to prematurity, making in turn preterm birth ranked as the second-leading reason for death for children who did not complete their fifth year and the number one fatal syndrome in the first month after birth. To suppress this mortality rate, a special unit known as the Neonatal Intensive Care Unit (NICU) is designed for premature babies care in hospitals. The health of the baby is maintained and monitored through this unit that encloses various types of monitors, and equipment utilized in different processes to achieve this objective [1]. The main instrument in NICU is the incubator; a closed system that supplies the infant with warmth [2]. Incubators are extremely expensive [3], it produces noise which affects the corona of the infant, this phenomena is known by Neurodevelopmental dungeon [3].
Also, incubator connect infant with electrodes continuously to measure the Electrocardiography (ECG), which may affect the infant since its skin is very weak [4]. Also, since the baby lies in a closed hood it is difficult to accesses the baby for medical procedure or even breast feeding [5].

Globally, prematurity is the leading cause of death in children. In high-income countries, almost all of these babies survive. In low-income settings, half of the babies born at 32 weeks ( 2 months early) or below die due to a lack of feasible, cost-effective care, such as warmth, breastfeeding support, and basic care for infections and breathing difficulties.

The aim of our project is to develop a new portable and wireless-base incubator. We tend to make it affordable, feasible, patient friendly and meet the health requirements for premature baby especially in low-income countries. The project focuses on the premature babies in the third trimester of pregnancy.

The paper is organized as follows. Section 2 presents the materials and methods utilized. Section 3 showcases the results obtained. Section 4 provides the discussion and conclusion of the work.

\section{Materials AND Methods}

The major steps of this study are summarized in the block diagram in Fig. 1. Fig. 1 showcases the biomedical system, which is the infant or preterm, the features, output and the decision making and possible actions whether temporary therapy or diagnosis.

\section{A. System Design}

Through our advanced incubator, three vital signs, heart rate, $\mathrm{SpO}_{2}$ and temperature, shown in Fig. 1, are continuously and wirelessly monitored in our work for a further diagnose and a temporary treatment in case of emergency. These vital signs are measured by ultra-low power noninvasive optical sensor based on infrared technology. Through a microcontroller, these signals are analyzed and reported on both a Liquid Crystal Display (LCD) mobile application, and hospital support web server. Furthermore, an emergency aid for any abnormal fluctuate in the infant's vital signs is supported, through controlling the heater, the oxygen release valve, or both simultaneously. The whole system is supported by either direct electricity supply or batteries. 


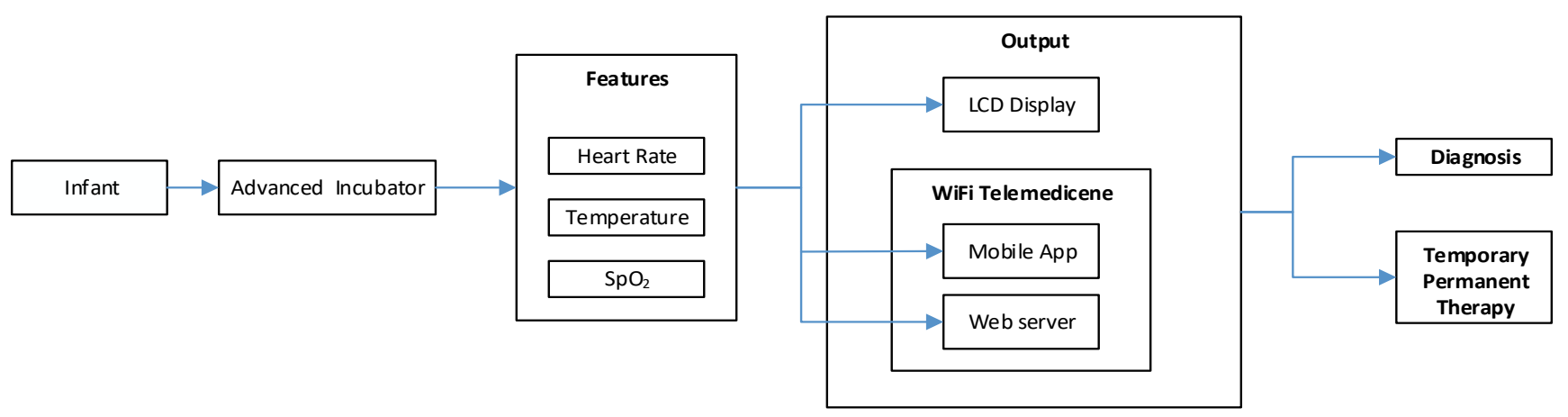

Fig. 1: Advanced Incubator Block Diagram.

\section{B. Extracted Features}

In this advanced project we extracted numerous features including the heart rate, Oxygen Saturation Percentage and temperature. These features are introduced in the upcoming subsections.

1) Heart Rate (HR): It represents the number of heart beats per minute. Electrical heart signals are commonly monitored through Electrocardiograph (ECG). ECG can analyze the heart signal by measuring its frequency, time domain, waveform and amplitude. Meanwhile, ECG requires continuous attachment of several electrodes with specific gel, which leads to discomfort, pain and damage of the fragile premature infant's skin. Besides, it is hard to be managed by the mother. To overcome this, we chose to extract the heart beats by an infrared technology.

2) Oxygen Saturation Percentage $\left(\mathrm{SpO}_{2}\right)$ : It is the arterial $\mathrm{SpO}_{2}$. In premature baby studies, there is a delicate balance between too much and too little supplemental oxygen exposures. Since overuse and under-use of supplemental oxygen can harm preemies, $\mathrm{SpO}_{2}$ must be monitored and kept within 90 to 93\% to avoid diseases. Pulse oximetry is an advantageous method of oxygenation monitoring, since it is continuous and noninvasive. In fact, in tiny premature babies, it is hard to draw sufficient amount of arterial blood frequently for a continuous measurement of the partial pressure of oxygen in arterial blood $\left(\mathrm{PaO}_{2}\right)$. Therefore, in our novel incubator we used a pulse oximetry [6].

3) Temperature (T): When infant's body temperatures are not regulated, they are at high risk of hypothermia. Neonatal hypothermia has been reported to rise up the risk of complications of preterm birth, coagulation abnormalities, infection etc. [7]. For that reason, we made sure to monitor the preemie's temperature continuously and non-invasively. Temperature was measured by means of a device comprising a sensor with a built in integrated circuit. In addition to a 5 thermistors embedded in gel sacks, to provide a feedback and avoid overheating or lower heating. Thermistors are variable resistors which changes its resistance as the temperature changes.

\section{Therapy Method}

Kumar et al. reported that babies beyond birth are exposed to a significant drop in their core and skin temperatures, due to the absence of subcutaneous fat that is no more metabolized into heat or utilized as an insulator [8]. Thereby, a warming system is utilized in our project. The warming system comprised two main parts: the phase change material and the heaters.

A continuous warm environment was established through gel packs, these gel packs preserve and transform the heat through conduction in order to provide the ideal required infant temperature. Cartridge Heaters of $12 \mathrm{~V}$ input and $40 \mathrm{~W}$ power were used for heating up the gel packs. The heater were supported with $9600 \mathrm{mAh}$ (milliamp.hour) lithium ion batteries. By referring to equations (1) and (2), the batteries can continuously turn the heater "on" to about 2.8 hours. To conserve a warm environment for the premature infant, heaters are periodically driven "ON" in order to avoid critical temperature drop or extra heating of infant's body. This temperature range accuracy is managed by a microcontroller.

$$
I=\frac{P}{V}
$$

where $I$ is the current, $P$ the power and $V$ the voltage.

$$
D=\frac{C}{I}
$$

where $D$ is the duration, $C$ the capacity and $I$ the current.

Heat loss is suppressed due to an isolation system which contains several types of fabrics. The isolation fabrics insulate the infant from outer environment and provide bio-compatible, breathable, antibacterial and smooth contact with infant skin. In addition to isolation fabrics, a plastic shield is embedded to ensure the safety of the infant by eliminating any mechanical load.

Moreover, an oxygen release part is implemented in our advanced incubator. This part is responsible for releasing oxygen when the infant needs an extra dosage of oxygen. Oxygen is released frontally above the premature infant's head. 


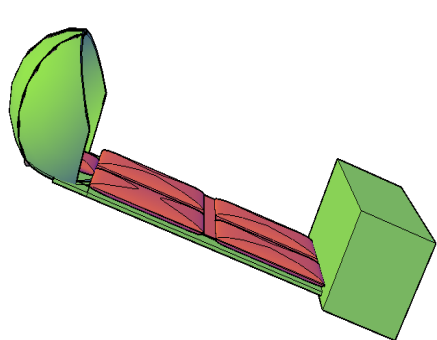

(a)

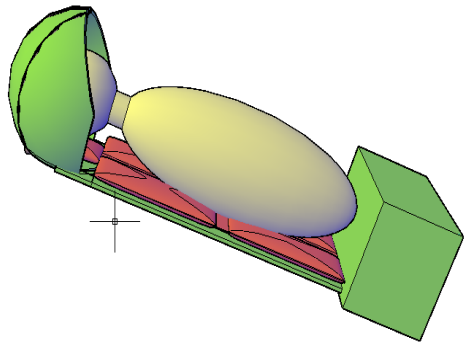

(b)
Fig. 2: Advanced Incubator Design. (a) The autocad of the system before placing the premature and (b) The autocad of the system after placing the premature.

The increased oxygen percentage in the atmosphere causes oxygen to diffuse into the lungs of the preemie.

\section{Output}

The user interface is designed to show up in two ways, a direct interface using LCD, and a Wireless Fidelity (Wi-Fi) data transmission through via web server, connected directly to the hospital doctors and a mobile application. This makes our system a Telemedicine supporting system.

\section{EXPERIMENTAL RESULTS}

To build the main blocks of the advanced incubator autocad simulation of the design was a requirement. This was associated with the system result and final design. Then to test the electronic components before connecting them to the desired design, these components especially the heaters and the power generator were tested. These were associated with the testing results due to their experimental application on heating water.

\section{A. System Results}

Fig. 2 represents our advanced incubator design in autocad. Our novel incubator is designed to be portable and easy to handle. In addition, the Advanced Incubator is designed to support the basic functions of an incubator by not only regulating the premature infant's body temperature, but also by monitoring some biological signals.

\section{B. Testing Results}

To test the performance of the constituents of our Advanced Incubator, the electronic components were tested and the results were reported in Figs. 3, 4, 5 and 6.

Fig. 3 showcases the variation of generated Power $(\mathrm{P})$ with respect to the voltage, at voltage $=0 \mathrm{~V}, \mathrm{a} P=0 \mathrm{~W}$. As voltage increase, power increase till it reach the maximum $\mathrm{P}=37 \mathrm{~W}$ at voltage equal $12 \mathrm{~V}$. A result proving that the power can be assigned through regulating the voltage.

Fig. 4 (a) reveals the variation of the thermistor resistance with respect to the temperature. A maximum resistance was recorded as $\mathrm{R}=190 \mathrm{k} \Omega$ at temperature $\mathrm{T}=-40^{\circ} \mathrm{C}$. According to Fig. 4 (a), as the temperature increases the resistance decreases until it reaches a minimum of $\mathrm{R}=0.7 \mathrm{k} \Omega$ at $\mathrm{T}=110^{\circ} \mathrm{C}$. In our advanced incubator, we were keen on measuring the variation of premature infant temperature at the room temperature. Both

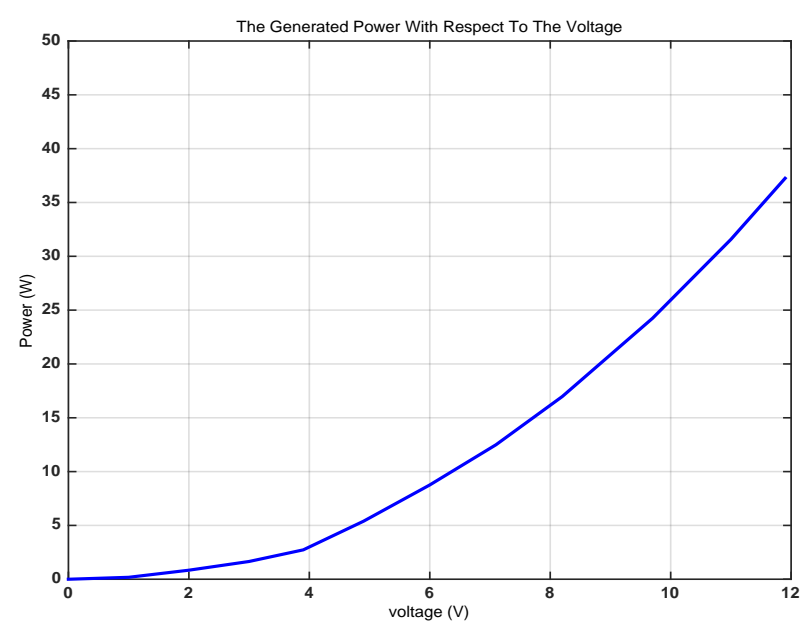

Fig. 3: The Generated Power in (W) Versus the Voltage in (V).

temperatures vary in a range between $20^{\circ} \mathrm{C}$ and $45^{\circ} \mathrm{C}$. In this range, the resistance decreases from $12.69 \mathrm{k} \Omega$ at $\mathrm{T}=20^{\circ} \mathrm{C}$ to $4.82 \mathrm{k} \Omega$ at $\mathrm{T}=25^{\circ} \mathrm{C}$. Since each sensor has to provide a variation of voltage, which is then converted to digital signal through an Analog to Digital Converter (ADC), we built a simple voltage divider circuit to convert the variation of the resistance into variation of the voltage.

Fig. 4 (b) showcases the variation of voltages with respect to the temperature. At $\mathrm{T}=20^{\circ} \mathrm{C}$, the output voltage was $V=2.8 \mathrm{~V}$, then it decreased as the temperature increased until it reached its minimum $V=1.6 \mathrm{~V}$ at $\mathrm{T}=45^{\circ} \mathrm{C}$.

Fig. 5 shows a variation of water temperature as a function of time. At $\mathrm{t}=0 \mathrm{~min}$ the initial temperature $\mathrm{T}=24{ }^{\circ} \mathrm{C}$, as time increases from $0.5 \mathrm{~min}$ till $6.5 \mathrm{~min}$ the temperature rises from $\mathrm{T}=24{ }^{\circ} \mathrm{C}$ till $\mathrm{T}=54{ }^{\circ} \mathrm{C}$. Over this duration, the heater converts 390 Joules (J) of electrical energy into thermal energy. This produced energy raises the temperature of $250 \mathrm{ml}$ of water from $\mathrm{T}=25^{\circ} \mathrm{C}$ till $\mathrm{T}=54{ }^{\circ} \mathrm{C}$.

Fig. 6 represents the drop down of temperature for $200 \mathrm{ml}$ of water at room temperature $25^{\circ} \mathrm{C}$ with and without thermal insulator. The effect of isolation is coded in red, and the effect of the absence of isolation is coded in blue. This curve shows that the temperature drops from $70 \Omega \mathrm{C}$ to $29 \Omega \mathrm{C}$ in $60 \mathrm{~min}$ without insulation. While with thermal insulation, it only drops from $70^{\circ} \mathrm{C}$ to $50^{\circ} \mathrm{C}$ within $60 \mathrm{~min}$. This indicates that, the thermal isolation can decrease the thermal lose and increase the time needed to drop temperature 6 times.

\section{DISCUSSION AND CONCLUSION}

The developed Advanced incubator promotes the existing infant incubators. In addition to the main function of the Advanced incubator such as warming, the incubator adds the mobility feature which makes the handling of the infant more easily, allows for breastfeeding and conserves the infantmother bond. The warming system consists of 3 main parts batteries, heaters, and insulation. Batteries are the sources of energy which give the system its mobility feature, it can 


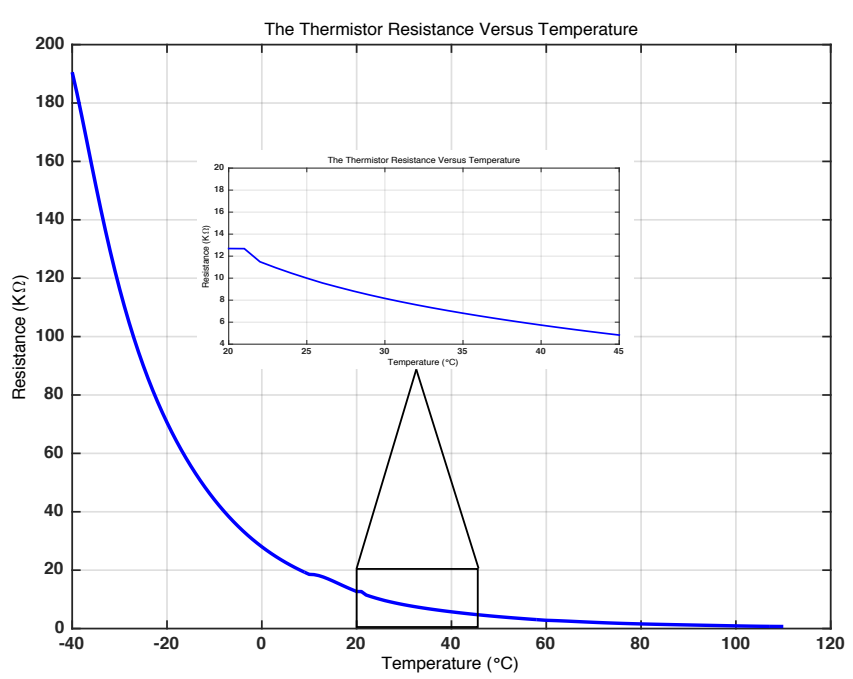

(a)

Fig. 4: Advanced Incubator Thermistor's Characteristics. (a) The Thermistor's Resistance Versus Temperature $\left({ }^{\circ} \mathrm{C}\right)$ and (b) The Thermistor's Voltage Versus Temperature $\left({ }^{\circ} \mathrm{C}\right)$.

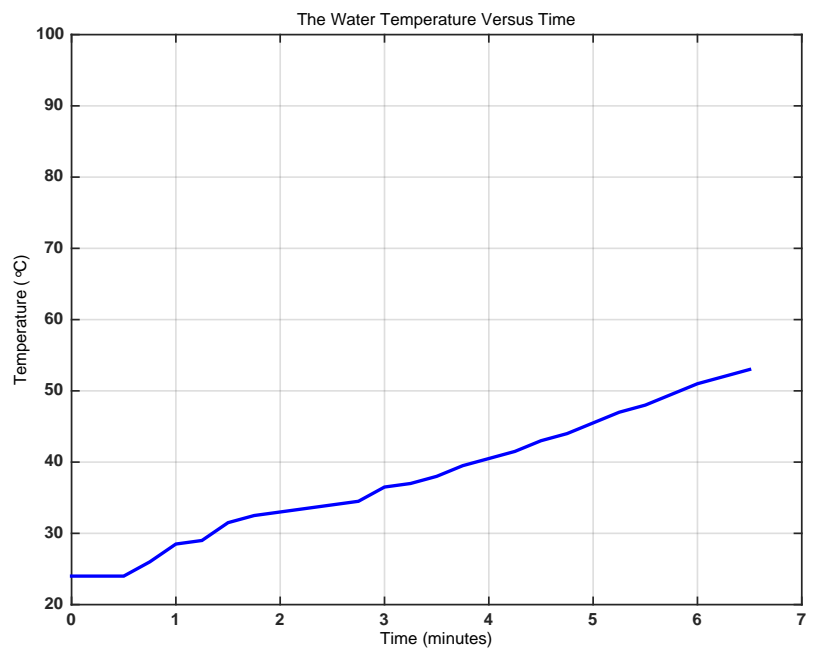

Fig. 5: The Water Temperature $\left({ }^{\circ} \mathrm{C}\right)$ Versus Time (minutes).

store enough energy to power the system. The stored energy is converted by a heater which acts as simple resistor that converts electrical energy into thermal energy. The last part is the isolation, it provides the use of the temperature that expands the batterie's life span.

To conclude, advanced incubator provides the warming feature of incubator, also it provides other features such as continuous monitoring and temporary treatment to get the healthcare necessary to the infant survive.

\section{REFERENCES}

[1] H. Blencowe, S. Cousens, M. Z. Oestergaard, D. Chou, A.-B. Moller, R. Narwal, A. Adler, C. V. Garcia, S. Rohde, L. Say et al., "National, regional, and worldwide estimates of preterm birth rates in the year 2010 with time trends since 1990 for selected countries: a systematic analysis and implications," The Lancet, vol. 379, no. 9832, pp. 2162-2172, 2012.

[2] M. Aita and L. Snider, "The art of developmental care in the nicu: A concept analysis," Journal of Advanced Nursing, vol. 41, no. 3, pp. 223232, 2003.

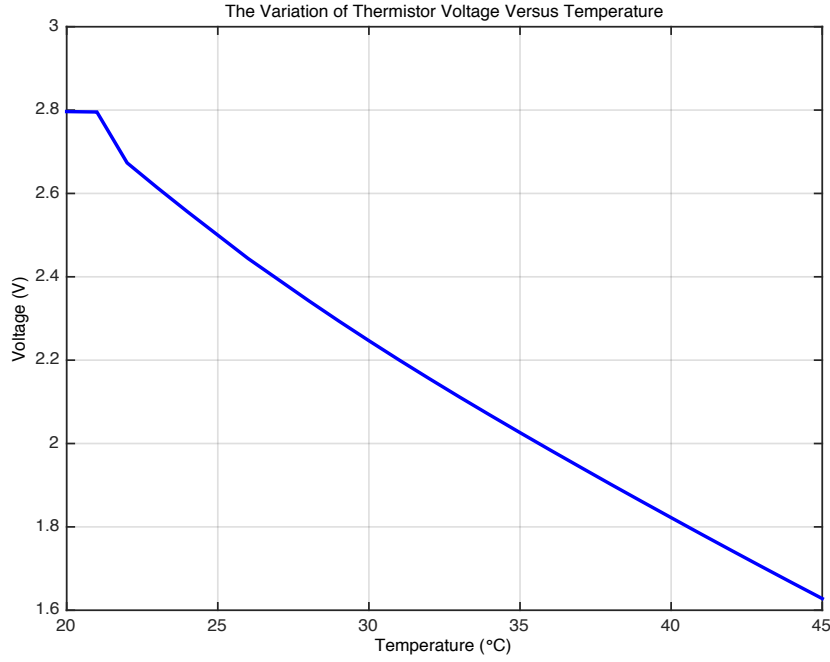

(b)

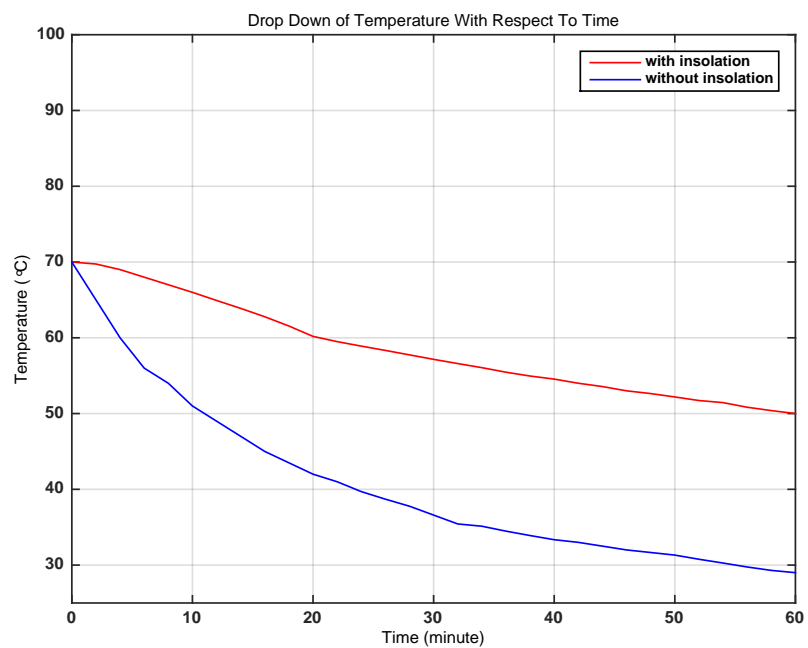

Fig. 6: The Water Temperature $\left({ }^{\circ} \mathrm{C}\right)$ Versus Time (minutes) With Isolation (Coded in Red) and Without Isolation (Coded in Blue).

[3] G. Blennow, N. W. Svenningsen, and B. Almquist, "Noise levels in infant incubators adverse effects," Pediatrics, vol. 53, no. 1, pp. 29-32, 1974.

[4] O. Bonner, K. Beardsall, N. Crilly, and J. Lasenby, "There were more wires than him: the potential for wireless patient monitoring in neonatal intensive care," BMJ Innovations, pp. bmjinnov-2016, Jan 2017.

[5] R. Maastrup, B. M. Hansen, H. Kronborg, S. N. Bojesen, K. Hallum, A. Frandsen, A. Kyhnaeb, I. Svarer, and I. Hallström, "Breastfeeding progression in preterm infants is influenced by factors in infants, mothers and clinical practice: the results of a national cohort study with high breastfeeding initiation rates," PloS one, vol. 9, no. 9, p. e108208, 2014.

[6] M. Chang, "Optimal oxygen saturation in premature infants," Korean journal of pediatrics, vol. 54, no. 9, pp. 359-362, Sep 2011.

[7] A. O. Lucas, B. J. Stoll, J. R. Bale et al., Improving birth outcomes: meeting the challenge in the developing world. National Academies Press, 2003.

[8] V. Kumar, J. Shearer, A. Kumar, and G. Darmstadt, "Neonatal hypothermia in low resource settings: a review," Journal of Perinatology, vol. 29 no. 6 , pp. 401-412, 2009. 